\title{
Evaluación de la dosis periférica en tratamientos de radiocirugía para blancos simulados en un fantoma
}

\author{
Evaluation of peripheral dose in radiosurgery treatments for \\ simulated targets in a phantom
}

\author{
Cecilia Querebalú́ ${ }^{*}$, Carlos Solano², Fernando Marquez ${ }^{3}$ \\ ${ }^{1}$ Instituto de Radiocirugía del Perú. Av. Universitaria 854, San Miguel, Lima, Perú \\ ${ }^{2}$ Facultad de Ciencias, Universidad Nacional de Ingeniería, Av. Túpac Amaru 210, Rímac, Lima, Perú \\ 3 Instituto Nacional de Enfermedades Neoplásicas, Av. Angamos Este 2520, Surquillo, Lima, Perú \\ Recibido (Recieved): 04/09/2018 Aceptado (Accepted): 13/12/2018
}

\section{RESUMEN}

En tratamientos de radiocirugía siempre hay una dosis absorbida, $D$, que se deposita fuera del campo de tratamiento, a esta $D$ se le conoce como "Dosis Periférica", DP. Hay una necesidad de optimizar el procedimiento para reducir los riesgos futuros relacionados a la DP. El objetivo de este trabajo fue evaluar la DP en 9 posiciones anatómicas, estás fueron, ojos, tiroides, parte superior de los pulmones, mediastino, mamas y ombligo de un fantoma antropomórfico. El desarrollo del trabajo contemplo el uso de películas radiocrómicas EBT2 y un haz de fotones de $6 \mathrm{MV}$ generados en un acelerador lineal Clinac 2100C/D. Analizando la influencia del tamaño de colimador, se encontró mayor DP $(150.81 \pm 0.97)$ cGy en el ojo derecho del fantoma para el colimador de $3 \mathrm{~cm}$ con un blanco posicionado en el plano superior derecho de la cabeza del fantoma y su influencia con la distancia de (27.14 \pm 1.0 ) CGy en la tiroides, para un blanco posicionado en el plano inferior izquierdo de la cabeza del fantoma para una $D$ prescrita de $18 \mathrm{~Gy}$. Se concluye que la DP reproduce una dependencia proporcional con respecto a las unidades de monitor, así mismo mientras más grande el colimador mayor es la DP, además haces de fotones que entran y salen del blanco en técnicas como la utilizada, que es la de arcos no coplanares, conlleva a que un mayor volumen de tejido sano este expuesto a recibir $D$ bajas.

Palabras Clave: radiocirugía, dosis periférica, películas radiocrómicas EBT2.

\section{ABSTRACT}

In radiosurgery treatments, there is always an absorbed dose, $D$, which is deposited outside the treatment field, this $D$ is known as "Peripheral Dose", PD. There is a need to optimize the procedure to reduce future risks related to PD. The objective of this work was to evaluate the PD in 9 anatomical positions, these were, eyes, thyroid, upper lungs, mediastinum, breasts and navel of an anthropomorphic phantom. The development of the work contemplates the use of the EBT2 radiochromic films and a photon beam of $6 \mathrm{MV}$ generated in a linear accelerator Clinac $2100 \mathrm{C} / \mathrm{D}$. Analyzing the influence of collimator size, greater PD $(150.81 \pm 0.97) \mathrm{cGy}$ was found in the right eye of the phantom for the collimator of $3 \mathrm{~cm}$ with a target fixed in the upper right plane of the head of the phantom and its influence with the distance of, a PD of $(27.14 \pm 1.0)$ cGy in the thyroid, for a target positioned in the lower left plane of the phantom head for a prescribed $D$ of $18 \mathrm{~Gy}$. It is concluded that the PD reproduces a proportional dependence with respect to the monitor units, likewise the larger the larger collimator is the PD, in addition to photons that enter and leave the target in techniques such as the one used, which is that of non-coplanar arcs, leads to a greater volume of healthy tissue being exposed to low $D$.

Keywords: radiosurgery, peripheral dose, EBT2 radiochromic films.

\section{INTRODUCCIÓN}

En radiocirugía se entregan tratamientos con altas $D$ por fracción, 10-20 Gy, a pesar de que en este procedimiento se tratan pequeños volúmenes y la $D$ es altamente localizada a cubrir el blanco y la caída de $D$ afuera de este es muy rápida, la DP es un considerable problema dosimétrico. Hablamos de DP, cuando cierta porción de la radiación es depositada fuera del campo de radiación. Las componentes de la DP son fuga del cabezal del acelerador lineal, linac, radiación dispersa de los colimadores y radiación dispersa dentro del paciente. Los dos primeros dependen de la técnica, equipo empleado y su diseño. La última depende de la energía de radiación, la distancia de los bordes al campo y en menor grado del tamaño de campo [1]. Esta D sería

* Corresponding author.:

E-mail: querebalu.cecilia@gmail.com 
considerable determinar en pacientes de larga esperanza de vida en donde los efectos secundarios podrían manifestarse clínicamente. En algunos estudios como los de Xu et al. [2], muestran evidencia del riesgo de aparición de tumores secundarios radioinducidos debido a la DP. Así mismo, Bonato, CC et al. [3], evaluaron DP con dosimetría in vivo en tiroides, en niños y adolescentes que recibieron radioterapia en diferentes sitios, teniendo como antecedente que $D$ tan bajas como 10 cGy están asociados con una mayor incidencia de nódulos tiroideos y cáncer de tiroides.

A partir de estos estudios y muchos más, donde estiman que estás DP deberían ser lo más bajas posibles [4], surge la pregunta, ¿en qué medida la evaluación de DP en tratamientos de radiocirugía con linac Clinac 2100 C/D con técnica de cuatro arcos y criterios de planificación, como tamaño del colimador, posición del blanco, unidades de monitor (UM), influye en la optimización de los tratamientos, y en la mejora de la planificación del tratamiento antes de que el paciente sea tratado?, sabiendo que epidemiológicamente hay evidencia de un incremento de riesgo de desarrollar cáncer después de largo tiempo de exposición a $D$ totales más altas que 50-100 mSv o exposición aguda a D más altas que 10-50 mSv. He aquí la importancia de reducir la DP tan bajo como sea posible, teniendo en cuenta que no hay un umbral de $D$ para efectos estocásticos [5].

Para ello, en este trabajo se evaluó la DP en nueve posiciones anatómicas de un fantoma antropomórfico de medio cuerpo: ojos, tiroides, parte superior de los pulmones, mediastino, mamas y ombligo, simulando blancos en toda la región del cerebro del fantoma, utilizando como dosímetros películas radiocrómicas, PRC, EBT2 [6]. Por ende se dividió el cerebro en 4 planos, para tratar lesiones que abarquen todo el encéfalo, en un fantoma con dimensiones recomendadas por la ICRP 89 [7] (para un niño de 8 años), y ubicar lesiones intracraneales de diferentes tamaños, y así evaluar la DP con el objetivo de que antes de cada tratamiento de radiocirugía se considere o se tenga conocimiento de la $D$ depositada fuera del volumen de tratamiento y de ser posible tomar precauciones para minimizar la DP. De esta manera, se cumplirá con una parte fundamental de un Programa de Garantía de Calidad en Radioterapia, cuyo objetivo primordial es implantar las normas y criterios de calidad para asegurar la optimización del tratamiento de radioterapia y la protección radiológica del paciente.

\subsection{Radiocirugía con linac}

Los tratamientos de radiocirugía consisten en dar una alta $D$ de radiación por fracción a un denominado blanco. La $D$ de radiación se prescribe en la curva que une puntos de igual dosis, isodosis, que cubre el blanco protegiendo el tejido que está alrededor. Para poder realizar estos tratamientos, en principios de 1980 se adaptaron linac para producir arcos de radiación colimados y enfocados con una alta tasa de radiación. Con los linac, la radiación es apuntada en un área específica del cuerpo, donde está el blanco o área a tratar. En el área esta un punto de referencia llamado isocentro, y es un punto donde se cruzan el eje de giro del brazo con el eje de giro del colimador y con el eje de giro de la mesa de tratamiento. Es así que con los linac adaptados para radiocirugía se entrega un haz de radiación colimado mientras esté rota alrededor del blanco. El tratamiento con linac puede aplicarse a blancos con diámetro entre 5 y $35 \mathrm{~mm}$. Para irradiar blancos de esos diámetros, se adapta un colimador terciario al linac y un sistema de fijación a la mesa, como se ilustra en la Figura 1.

Hay que tener en cuenta que la radiocirugía se toma en cuenta cuando se tienen lesiones intracraneales que son consideradas inoperables, o cuando existe un riesgo excesivo si se aplica una cirugía convencional [8].

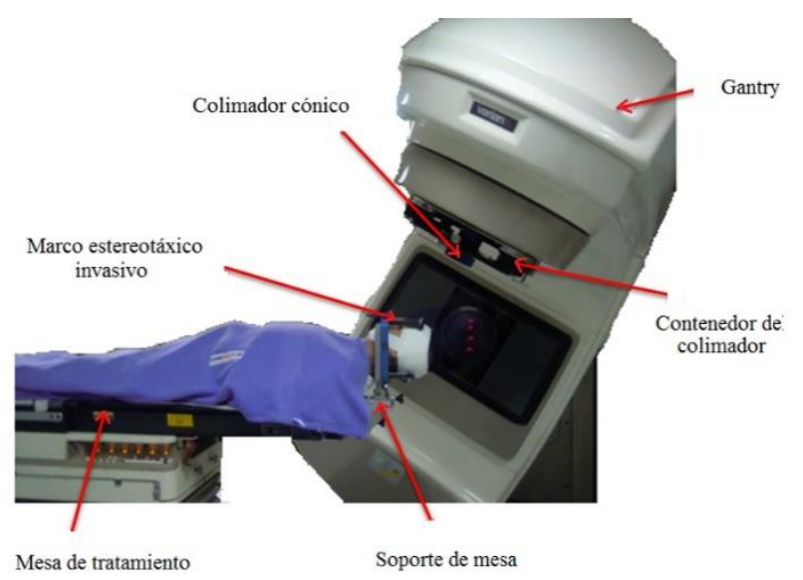

Figura 1. Acelerador lineal Varian Clinac 2100 C/D, adaptado para radiocirugía.

\subsection{Películas radiocrómicas EBT2}

Las películas radiocrómicas, PRC, han sido utilizadas para fines dosimétricos desde 1960, especialmente para medida de $D$ en haces de electrones [9]. Con el avance de la tecnología hasta estos años, ha hecho posible que la dosimetría con PRC tenga mayores ventajas, estas son: composición cercana al tejido equivalente, alta resolución espacial, alto rango de $D$, relativamente baja dependencia energética, insensible a la luz visible (aunque exhiben algo de sensibilidad a la luz ultravioleta y temperatura) y sin necesidad de proceso químico. La PRC que se utilizó en este trabajo, es la gafchromic EBT2, está PRC está compuesta de dos láminas de poliéster que cubre la capa activa y la adhesiva, tal como lo ilustra la Figura 2. Las láminas de poliéster ayudan a proteger la capa activa del daño mecánico que puedan sufrir las películas en el cortado o en su manipulación, así mismo permite sumergirlas en agua por algunas horas (a costo de una penetración de agua de 1 a $2 \mathrm{~mm}$ de los bordes) y la capa que contiene 
la componente activa es la que se polimeriza de forma parcial cambiando de transmitancia (volviéndose más oscura a la luz visible). Después de que la PRC se haya expuesto a la radiación, esta se volverá más opaca mientras conforme aumente la $D$ recibida $[10,11]$.

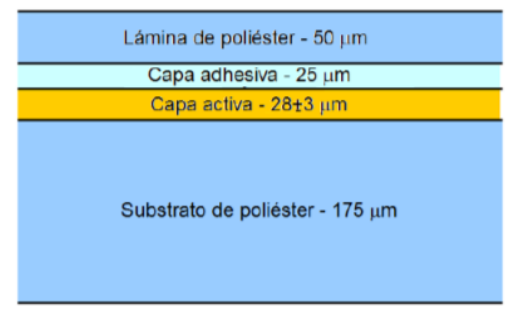

Figura 2. Corte transversal de una película radiocrómica EBT2 [10].

La transmisión de luz a través de la PRC puede ser medida con sistemas de escaneo densitométrico, estos son: densitómetro, escáner, espectrofotómetro, fuentes de luz, detectores de luz. Aquí es donde termina el proceso dosimétrico [12]. La transmisión de luz es una función de la opacidad de la película y puede ser medido en términos de densidad óptica, DO. Para poder elegir un sistema de escaneo, se debe evaluar el espectro de este, de tal manera que tenga una respuesta espectral relacionada con la absorbancia de la película.

\section{MATERIALES Y MÉTODOS}

Para la realización de este trabajo se utilizó el linac Varian Clinac 2100C/D (Varian Medical Systems de Palo Alto, California, EE. UU.) de la clínica San Pablo, Lima; el cual ha sido adaptado para realizar radiocirugía por el Instituto de radiocirugía del Perú (INRAPE). A continuación, se describe los materiales y la metodología desarrollada en el trabajo.

\subsection{Procedimiento de escaneo}

Para digitalizar la PRC se hizo uso de un escáner. El que se usó, es el Epson Expression 11000 XL [13]. La Figura 3 ilustra el escáner con PRC. Este es un escáner de imagen a color tipo flatbed equipado con una unidad de transparencia para películas, mide las componentes en color rojo, verde y azul de la PRC en una profundidad de color de 16 bits por canal. Tiene una respuesta espectral que está en relación con la absorbancia de la PRC EBT2 [14, 15].

Se escanearon las PRC siguiendo el protocolo dado por Devic et al. [16], para ello, se escanearon las piezas de PRC 5 veces antes y después de irradiarlas. Así se obtiene la lectura de transmisión del escáner como las desviaciones estándar, estos valores fueron determinados para cada pieza de PRC como un valor de pixel promedio sobre el ROI (región de interés) elegido. Además, se escaneo 5 veces una cartulina o pieza opaca negra (en este trabajo se usó piezas de película doi: https://doi.org/10.21754/tecnia.v28i2.343 radiográfica Oncology EC Film de Carestream [17], expuestas a luz y posteriormente reveladas), para obtener el valor de intensidad transmitida de luz cero, la cual caracteriza la señal de fondo del escáner, así como su correspondiente desviación estándar.

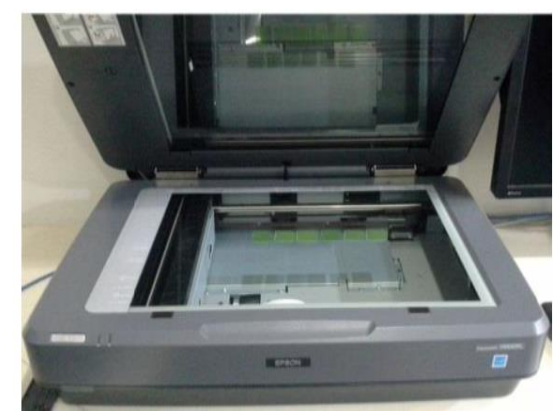

Figura 3. Escáner Epson Expression 11000 XL con PRC en su superficie.

La ecuación (1) y (2) muestra como se calcula la DO $_{\text {neta }}$ para una dosis $D_{j}$ :

$\mathbf{D O}_{\text {neta }}\left(D_{j}\right)=\mathbf{D O}_{\text {irrad. }}\left(D_{j}\right)-\mathbf{D O}_{\text {no irrad. }}\left(D_{j}\right)$

$\mathrm{DO}_{\text {neta }}\left(D_{j}\right)=\log _{10}\left(\frac{\mathrm{VP}_{\text {no irrad. }}-\mathrm{VP}_{\text {fondo }}}{\mathrm{VP}_{\text {irrad. }}-\mathrm{VP}_{\text {fondo }}}\right)$

donde $\mathbf{V P}_{\text {no irrad. }}, \mathbf{V P}_{\text {irrad. }}$ y $\mathbf{V P}_{\text {fondo }}$, son los valores de pixel promedio del ROI de las piezas de PRC no irradiada, irradiadas y sin transmisión de luz (película radiográfica EC Film) respectivamente.

Las lecturas de transmisión de escáner, $\mathbf{V P}_{\text {no irrad. }} \mathbf{O} \mathbf{V P}_{\text {irrad. }}$ así como las desviaciones estándares $\boldsymbol{\sigma}_{\mathrm{VP}_{\text {no irrad. }}} \mathbf{0} \boldsymbol{\sigma}_{\mathrm{VP}_{\text {irrad. }}}$, fueron determinadas sobre el mismo ROI para cada pieza de PRC. $Y$ valores para $\mathbf{V P}_{\text {fondo }} \mathrm{y}$ $\boldsymbol{\sigma}_{\text {fondo }}$ se determinaron como medias ponderadas de las cinco piezas de la película opaca simulada (sin transmisión de luz). Por otro lado la incertidumbre de la DOneta para una dosis $D_{j}$ se obtuvo de la ecuación (3).

$\frac{1}{\ln 10} \sqrt{\frac{\left(\sigma_{\mathrm{VP}_{\text {no irrad. }}}\left(D_{j}\right)\right)^{2}+\left(\sigma_{\text {fondo }}\right)^{2}}{\left(\mathrm{VP}_{\text {no irrad. }}\left(D_{j}\right)-\mathrm{VP}_{\text {fondo }}\right)^{2}}+\frac{\left(\sigma_{\mathrm{VP}_{\text {irrad. }}}\left(D_{j}\right)\right)^{2}+\left(\sigma_{\text {fondo }}\right)^{2}}{\left(\mathrm{VP}_{\text {irrad. }}\left(D_{j}\right)-\mathrm{VP}_{\text {fondo }}\right)^{2}}(3)}$

\subsection{Descripción de la técnica de radiocirugía}

Tabla 1 muestra la técnica de tratamiento que se utilizó en este trabajo, la cual fue de 4 arcos multiples no coplanares, con una amplitud de los arcos de $120^{\circ}$ con camilla estacionaria.

TABLA 1. Técnica de tratamiento utilizada en radiocirugía con 4 arcos, con angulación de $120^{\circ}$.

\begin{tabular}{cccc}
\hline \multirow{2}{*}{$\begin{array}{c}N^{\circ} \text { de } \\
\text { arco }\end{array}$} & \multicolumn{2}{c}{ Ángulo de gantry } & \multirow{2}{*}{\begin{tabular}{c} 
Ángulo \\
\cline { 2 - 3 } de camilla
\end{tabular}} \\
\hline 1 & $210^{\circ}$ & $330^{\circ}$ & $-75^{\circ}$
\end{tabular}

Revista TECNIA Vol. 28 N 1 Agosto - Diciembre 2018 


\begin{tabular}{llll}
2 & $330^{\circ}$ & $210^{\circ}$ & $-45^{\circ}$ \\
3 & $30^{\circ}$ & $150^{\circ}$ & $+45^{\circ}$ \\
4 & $150^{\circ}$ & $30^{\circ}$ & $+75^{\circ}$ \\
\hline
\end{tabular}

\subsection{Obtención de las imágenes tomográficas del fantoma y planificación del tratamiento}

Figura 4 ilustra a la izquierda el fantoma con el inmovilizador y las distancias a los puntos a medir la DP, ojos, tiroides, parte superior de los pulmones (como referencia se tomara la parte media de las clavículas), mediastino (como referencia se tomara la parte media del esternón), mamas y ombligo. Y a la derecha 3 cortes (sagital, axial y coronal) obtenidos de la tomografía y la reconstruccion 3D de la cabeza del fantoma obtenidas por el sistema de planificación de tratamiento, SPT, Simuplan.

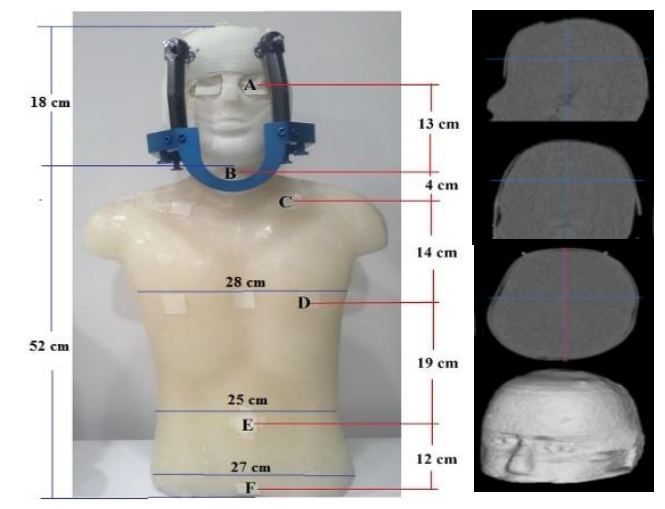

Figura 4. A la izquierda dimensiones del fantoma utilizado para la medida de DP y a la derecha cortes sagital, axial y coronal y reconstrucción 3D obtenidas con el SPT.

En el SPT se simularon blancos en distintas posiciones de la cabeza del fantoma dividiéndola en 4 planos, tal como lo ilustra la Figura 5. Los dos primeros planos se dividieron en 4 partes (inferior derecho, inferior izquierdo, superior derecho, y superior izquierdo), el tercer plano solo se dividió en 2 partes (superior derecho y superior izquierdo), y el cuarto plano se ubicó en el centro.

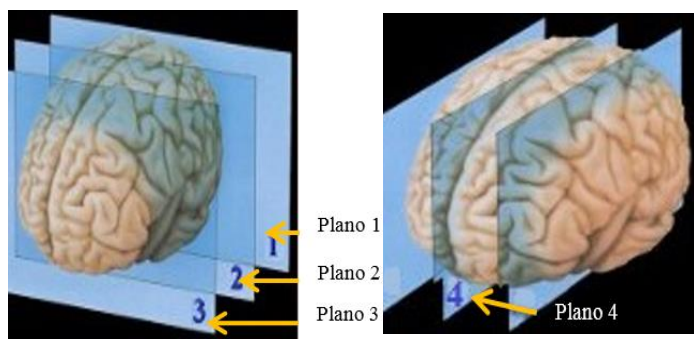

Figura 5. Imágenes reimpresas para mostrar la división dı

$$
\text { planos [9]. }
$$

Figura 6 muestra imágenes reimpresas y adaptadas para apreciar la división de los planos en los tres cortes (sagital, coronal y axial). Cabe mencionar que se realizaron 57 planes de radiocirugía con tamaños de conos de 1,2 y $3 \mathrm{~cm}$ de diámetro.

doi: https://doi.org/10.21754/tecnia.v28i2.343

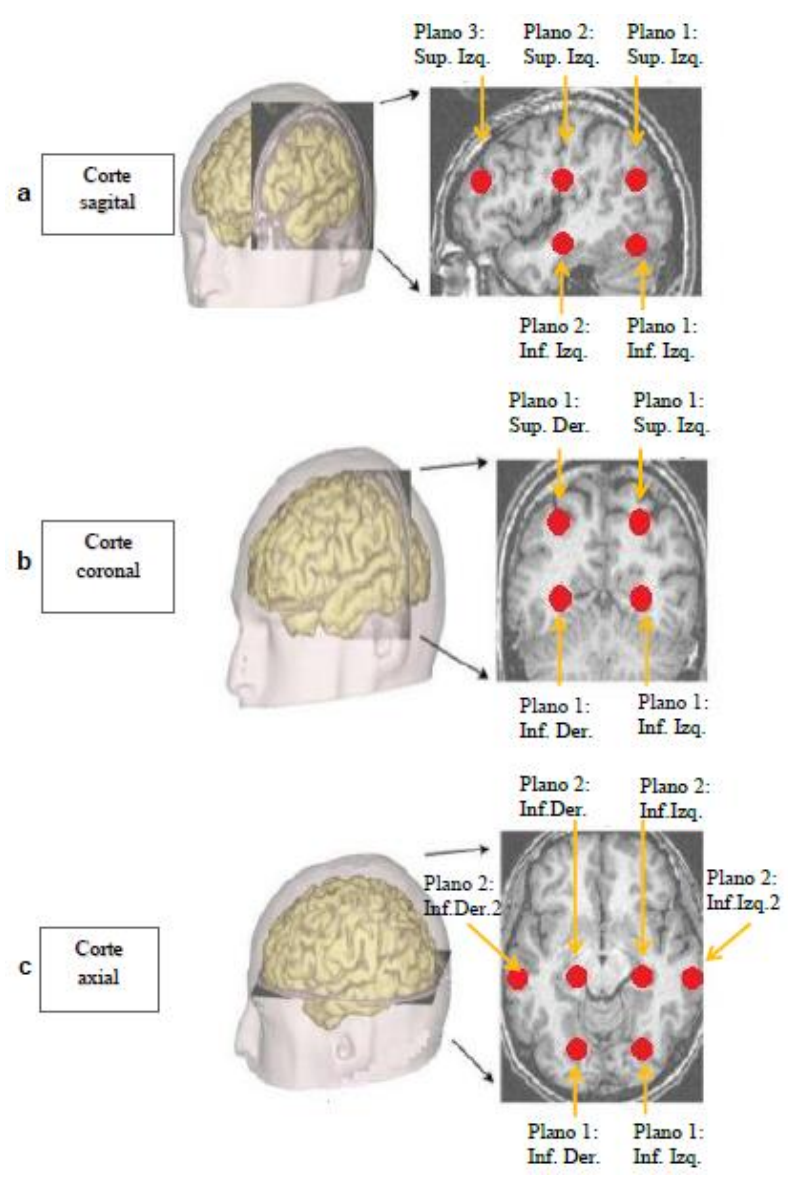

Figura 6. Imágenes reimpresas y adaptadas para mostrar la división de los planos en 3 cortes: a) sagital, b) coronal y c) axial [18]

\section{RESULTADOS}

\subsection{Caracterización y calibración de las PRC}

En la caracterización de las PRC, se encontró que la reproducibilidad de la respuesta de las $P R C$ es de $\pm 1.3 \%$ (24) para una D aplicada de 47.4 CGy; un coeficiente de linealidad de $r^{2}=0.9998$ para un rango de D de 1-93 cGy;

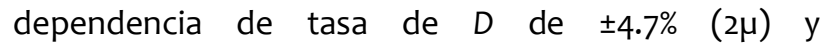
comportamiento lineal con coeficiente de correlación de 0.9412 en respuesta energética para energías en un linac de $6 \mathrm{MV}, 18 \mathrm{MV}$ y para un ${ }^{60} \mathrm{Co}$ para una $\mathrm{D}$ aplicada de $10 \mathrm{cG}$. Finalmente la Figura 7 ilustra la curva de calibración de las PRC EBT2 en un rango de $D$ de 0-85.2 cGy para una energía de $6 \mathrm{MV}$, encontrándose una máxima incertidumbre de $1.8 \%(2 \mu)$.

De la curva de calibración se encontró la ecuación de ajuste, ecuación (4), siendo está una polinómica de tercer grado.

$$
\mathrm{DO}_{\text {neta }}(D)=a D^{3}+b D^{2}+\mathrm{c} D+d
$$

donde

$\mathrm{DO}_{\text {neta }}$ es la densidad óptica neta;

$D \quad$ es la dosis absorbida (cGy). 


\section{Querebalú et al.}

Los coeficientes de la ecuación (4) se muestran en la Tabla 2.

TABLA 2. Coeficientes utilizados en la ecuación (4).

\begin{tabular}{lc}
\hline \multicolumn{2}{c}{ Coeficientes } \\
\hline a & $-1.06 \times 10^{-8}$ \\
b & $-6.96 \times 10^{-7}$ \\
c & $1.02 \times 10^{-3}$ \\
d & $-3.47 \times 10^{-5}$ \\
\hline
\end{tabular}

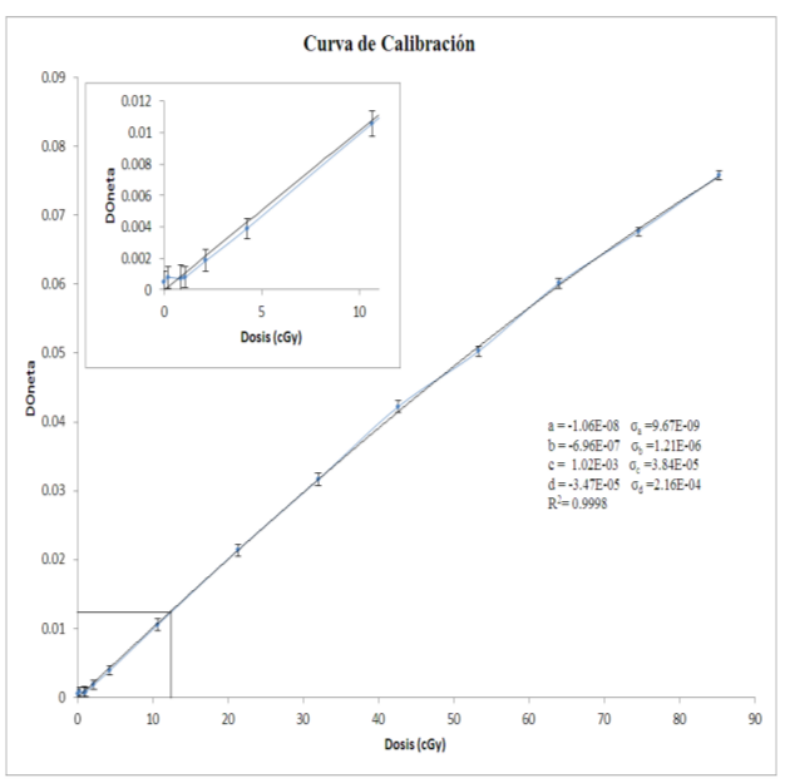

Figura 7. Curva de calibración para la PRC EBT2, para una energía de $6 \mathrm{MV}$ en un rango de dosis de 0-85.2 cGy.

\subsection{Dosis periférica en las zonas de interés en el fantoma}

Las siguientes tablas muestran la DP en 9 posiciones del fantoma, para tamaños de conos de $1 \mathrm{~cm}, 2 \mathrm{~cm}$ y 3 $\mathrm{cm}$ de diámetro en los 4 planos. Para el plano 1 tenemos la Tabla 3, Tabla 4, Tabla 5 y Tabla 6, todas para una prescripción de D de 18 Gy al 80\% de isodosis. Para el plano 2 tenemos la Tabla 7, Tabla 8, Tabla 9, Tabla 10, Tabla 11 y Tabla 12 todas para una prescripción de $D$ de 18 Gy al 80\% de iodosis. Para el plano 3 tenemos la Tabla 13 y la Tabla 14, ambas para una prescripción de $D$ de 18 Gy al $80 \%$ de isodosis. Finalmente en el plano 4 tenemos la Tabla 15, Tabla 16 y Tabla 17, para una prescripción de D de 18 Gy al 80\%, 12 Gy al 80\% y 12 Gy al 50\% de isodosis respectivamente.

TABLA 3. DP en el Plano 1: Inf. Der. con prescripción de D de 18 Gy al $80 \%$ de isodosis

\begin{tabular}{lccc}
\hline Plano 1: Inf. Der. & \multicolumn{3}{c}{$18 \mathrm{~Gy}$ al 80\% de isodosis } \\
\hline Cono & $1.0 \mathrm{~cm}$ & $2.0 \mathrm{~cm}$ & $3.0 \mathrm{~cm}$ \\
\hline UM & 3796 & 3136 & 2920 \\
\hline Posición & \multicolumn{3}{c}{ Dosis periférica (cGy) } \\
\hline Ojo Der. & $5.97 \pm 0.54$ & $9.38 \pm 1.3$ & $10.20 \pm 0.92$
\end{tabular}

doi: https://doi.org/10.21754/tecnia.v28i2.343

$\begin{array}{lccc}\text { Ojo Izq. } & 4.13 \pm 0.61 & 7.07 \pm 1.5 & 8.22 \pm 1.0 \\ \text { Tiroides } & 4.39 \pm 0.64 & 10.66 \pm 1.2 & 17.91 \pm 1.3 \\ \text { Pulmón Sup. Der. } & 6.63 \pm 1.0 & 15.02 \pm 1.2 & 21.49 \pm 1.2 \\ \text { Pulmón Sup. Izq. } & 2.58 \pm 0.44 & 8.03 \pm 1.3 & 8.98 \pm 1.1 \\ \text { Mama der. } & 2.02 \pm 0.77 & 3.34 \pm 1.2 & 5.53 \pm 0.74 \\ \text { Mama izq. } & 1.65 \pm 0.77 & 2.73 \pm 1.2 & 4.05 \pm 0.69 \\ \text { Esternón } & 2.54 \pm 0.98 & 3.59 \pm 1.1 & 5.59 \pm 0.65 \\ \text { Ombligo } & 1.47 \pm 1.0 & 1.85 \pm 0.86 & 2.12 \pm 0.64\end{array}$

TABLA 4. DP en el Plano 1: Inf. Izq. con prescripción de D de 18 Gy al $80 \%$. de isodosis.

\begin{tabular}{lccc}
\hline Plano 1: Inf. Izq. & \multicolumn{3}{c}{18 Gy al 80\% de isodosis } \\
\hline Cono & $1.0 \mathrm{~cm}$ & $2.0 \mathrm{~cm}$ & $3.0 \mathrm{~cm}$ \\
\hline UM & 3756 & 3104 & 2892 \\
\hline Posición & \multicolumn{3}{c}{ Dosis periférica (cGy) } \\
\hline Ojo Der. & $4.33 \pm 1.0$ & $6.26 \pm 0.61$ & $8.41 \pm 0.71$ \\
Ojo Izq. & $5.00 \pm 1.1$ & $7.37 \pm 0.51$ & $9.79 \pm 0.80$ \\
Tiroides & $5.21 \pm 1.1$ & $10.89 \pm 0.58$ & $18.89 \pm 0.70$ \\
Pulmón Sup. Der. & $3.35 \pm 1.5$ & $4.87 \pm 0.47$ & $5.42 \pm 1.8$ \\
Pulmón Sup. Izq. & $4.09 \pm 1.3$ & $10.21 \pm 0.82$ & $21.65 \pm 0.61$ \\
Mama der. & $1.21 \pm 0.95$ & $1.45 \pm 0.43$ & $1.69 \pm 1.6$ \\
Mama izq. & $2.08 \pm 0.87$ & $2.63 \pm 0.70$ & $2.96 \pm 1.6$ \\
Esternón & $2.10 \pm 0.89$ & $2.76 \pm 0.70$ & $3.45 \pm 1.7$ \\
Ombligo & $1.02 \pm 0.80$ & $1.43 \pm 0.65$ & $2.04 \pm 1.7$ \\
\hline
\end{tabular}

TABLA 5. DP en el Plano 1: Sup. Der. con prescripción de D de 18 Gy al $80 \%$ de isodosis.

\begin{tabular}{|c|c|c|c|}
\hline \multicolumn{4}{|c|}{ sodos } \\
\hline Cono & $1.0 \mathrm{~cm}$ & $2.0 \mathrm{~cm}$ & $3.0 \mathrm{~cm}$ \\
\hline UM & 3092 & 2588 & 2440 \\
\hline Posición & \multicolumn{3}{|c|}{ Dosis periférica (cGy) } \\
\hline Ojo Der. & $5.25 \pm 0.74$ & $5.93 \pm 1.3$ & $11.04 \pm 0.83$ \\
\hline Ojo Izq. & $12.38 \pm 0.85$ & $18.68 \pm 1.3$ & $30.31 \pm 0.92$ \\
\hline Tiroides & $2.23 \pm 0.71$ & $3.12 \pm 0.51$ & $6.59 \pm 0.87$ \\
\hline Pulmón Sup. Der. & $5.62 \pm 0.69$ & $7.41 \pm 0.63$ & $14.27 \pm 0.90$ \\
\hline Pulmón Sup. Izq. & $2.04 \pm 0.75$ & $2.71 \pm 0.53$ & $5.23 \pm 0.95$ \\
\hline Mama der. & $1.30 \pm 0.82$ & $1.63 \pm 0.59$ & $2.17 \pm 0.90$ \\
\hline Mama izq. & $0.92 \pm 0.98$ & $2.14 \pm 0.53$ & $5.17 \pm 0.97$ \\
\hline Esternón & $0.66 \pm 1.0$ & $1.10 \pm 0.56$ & $1.65 \pm 1.1$ \\
\hline Ombligo & $0.60 \pm 1.0$ & $0.92 \pm 0.85$ & $1.34 \pm 1.0$ \\
\hline
\end{tabular}

TABLA 6. DP en el Plano 1: Sup. Izq. con prescripción de D de 18 Gy al $80 \%$ de isodosis.

\begin{tabular}{lccc}
\hline Plano 1: Sup. Izq. & \multicolumn{3}{c}{$18 \mathrm{~Gy}$ al $80 \%$ de isodosis } \\
\hline Cono & $1.0 \mathrm{~cm}$ & $2.0 \mathrm{~cm}$ & $3.0 \mathrm{~cm}$ \\
\hline UM & 3064 & \multicolumn{3}{c}{ Dosis periférica (cGy) } \\
\hline Posición & $6.19 \pm 0.36$ & $10.53 \pm 0.77$ & $17.09 \pm 0.51$ \\
\hline Ojo Der. & $5.68 \pm 0.54$ & $6.94 \pm 0.82$ & $13.28 \pm 0.63$ \\
Ojo Izq. & $2.21 \pm 0.45$ & $4.60 \pm 0.78$ & $5.10 \pm 0.60$ \\
Tiroides & $2.19 \pm 0.58$ & $2.66 \pm 0.79$ & $3.32 \pm 0.73$ \\
Pulmón Sup. Der. & $6.24 \pm 0.58$ & $8.54 \pm 0.82$ & $12.80 \pm 0.78$ \\
Pulmón Sup. Izq. & $1.46 \pm 0.60$ & $1.52 \pm 0.81$ & $3.09 \pm 0.70$ \\
Mama der. & $0.68 \pm 1.2$ & $1.01 \pm 0.94$ & $2.16 \pm 0.93$ \\
Mama izq. & $0.29 \pm 1.3$ & $0.56 \pm 1.0$ & $1.79 \pm 0.96$ \\
Esternón & $0.23 \pm 1.2$ & $0.40 \pm 1.0$ & $1.48 \pm 0.93$ \\
Ombligo & & & \\
\hline
\end{tabular}

TABLA 7. DP en el Plano 2: Inf. Der. con prescripción de $D$ de 18 Gy al $80 \%$ de isodosis.

\begin{tabular}{lccc}
\multicolumn{4}{c}{$80 \%$ de isodosis. } \\
\hline Plano 2: Inf. Der. & \multicolumn{3}{c}{$18 \mathrm{~Gy}$ al $80 \%$ de isodosis } \\
\hline Cono & $1.0 \mathrm{~cm}$ & $2.0 \mathrm{~cm}$ & $3.0 \mathrm{~cm}$ \\
\hline UM & 3984 & 3284 & 3048 \\
\hline Posición & \multicolumn{3}{c}{ Dosis periférica (cGy) } \\
\hline Ojo Der. & $8.16 \pm 0.76$ & $12.26 \pm 0.68$ & $19.11 \pm 0.58$ \\
Ojo Izq. & $5.48 \pm 0.78$ & $9.97 \pm 0.62$ & $13.74 \pm 0.62$ \\
Tiroides & $6.57 \pm 0.85$ & $13.64 \pm 0.50$ & $20.44 \pm 0.78$
\end{tabular}

Revista TECNIA Vol. 28 N$^{\circ}$ Agosto - Diciembre 2018 
Pulmón Sup. Der. Pulmón Sup. Izq.

Mama der.

Mama izq.

Esternón

Ombligo

TABLA 8. DP en el Plano 2: Inf. Der. 2 con prescripción de D de 18 Gy al $80 \%$ de isodosis.

\begin{tabular}{lccc}
\hline Plano 2: Inf. Der .2 & \multicolumn{3}{c}{$18 \mathrm{~Gy}$ al $80 \%$ de isodosis } \\
\hline Cono & $1.0 \mathrm{~cm}$ & $2.0 \mathrm{~cm}$ & $3.0 \mathrm{~cm}$ \\
\hline UM & 3644 & 3020 & 2816 \\
\hline Posición & \multicolumn{3}{c}{ Dosis periférica (cGy) } \\
\hline Ojo Der. & $5.93 \pm 1.1$ & $8.28 \pm 0.76$ & $12.26 \pm 1.1$ \\
Ojo Izq. & $2.19 \pm 1.1$ & $4.37 \pm 0.82$ & $6.09 \pm 1.2$ \\
Tiroides & $3.46 \pm 1.1$ & $4.71 \pm 0.81$ & $8.95 \pm 1.3$ \\
Pulmón Sup. Der. & $3.62 \pm 1.1$ & $4.63 \pm 0.87$ & $8.83 \pm 0.64$ \\
Pulmón Sup. Izq. & $3.08 \pm 1.1$ & $4.19 \pm 0.91$ & $4.70 \pm 0.75$ \\
Mama der. & $1.62 \pm 1.0$ & $1.87 \pm 0.78$ & $2.12 \pm 0.94$ \\
Mama izq. & $1.12 \pm 1.0$ & $1.21 \pm 0.75$ & $1.98 \pm 0.81$ \\
Esternón & $0.93 \pm 1.0$ & $1.19 \pm 0.74$ & $1.78 \pm 0.95$ \\
Ombligo & $0.75 \pm 1.0$ & $1.09 \pm 0.72$ & $1.35 \pm 0.88$ \\
\hline
\end{tabular}

TABLA 9. DP en el Plano 2: Inf. Izq. con prescripción de D de 18 Gy al $80 \%$ de isodosis.

\begin{tabular}{lccc}
\hline \multicolumn{4}{c}{$80 \%$ de isodosis. } \\
\hline Cono & \multicolumn{3}{c}{$18 \mathrm{~Gy}$ al $80 \%$ de isodosis } \\
\hline UM & $1.0 \mathrm{~cm}$ & $2.0 \mathrm{~cm}$ & $3.0 \mathrm{~cm}$ \\
\hline Posición & 3976 & 3276 & 3044 \\
\hline Ojo Der. & \multicolumn{3}{c}{ Dosis periférica (cGy) } \\
Ojo Izq. & $5.36 \pm 1.1$ & $9.96 \pm 1.0$ & $14.80 \pm 0.81$ \\
Tiroides & $9.91 \pm 1.1$ & $12.19 \pm 0.69$ & $20.01 \pm 0.85$ \\
Pulmón Sup. Der. & $7.10 \pm 1.1$ & $13.08 \pm 0.71$ & $27.14 \pm 1.0$ \\
Pulmón Sup. Izq. & $6.96 \pm 1.2$ & $5.78 \pm 0.78$ & $8.48 \pm 0.99$ \\
Mama der. & $2.38 \pm 1.4$ & $12.59 \pm 0.80$ & $20.72 \pm 0.96$ \\
Mama izq. & $2.43 \pm 1.4$ & $3.40 \pm 0.81$ & $4.14 \pm 1.0$ \\
Esternón & $2.14 \pm 1.3$ & $3.13 \pm 0.75$ & $3.93 \pm 0.89$ \\
Ombligo & $1.74 \pm 1.4$ & $1.99 \pm 1.0$ & $2.11 \pm 0.90$ \\
\hline
\end{tabular}

TABLA 10. DP en el Plano 2: Inf. Izq. 2 con prescripción de D de 18 Gy al $80 \%$ de isodosis.

\begin{tabular}{lccc}
\hline Plano 2: Inf. Izq. 2 & \multicolumn{3}{c}{18 Gy al $80 \%$ de isodosis } \\
\hline Cono & $1.0 \mathrm{~cm}$ & $2.0 \mathrm{~cm}$ & $3.0 \mathrm{~cm}$ \\
\hline UM & 3624 & 3004 & 2804 \\
\hline Posición & \multicolumn{3}{c}{ Dosis periférica (cGy) } \\
\hline Ojo Der. & $2.15 \pm 1.0$ & $5.19 \pm 0.65$ & $8.56 \pm 064$ \\
Ojo Izq. & $7.17 \pm 1.1$ & $10.09 \pm 0.67$ & $20.08 \pm 0.87$ \\
Tiroides & $3.16 \pm 1.1$ & $5.31 \pm 0.60$ & $8.79 \pm 0.72$ \\
Pulmón Sup. Der. & $3.05 \pm 0.70$ & $3.36 \pm 1.3$ & $7.34 \pm 1.3$ \\
Pulmón Sup. Izq. & $3.52 \pm 0.78$ & $4.62 \pm 1.4$ & $11.05 \pm 0.75$ \\
Mama der. & $1.12 \pm 0.78$ & $1.56 \pm 1.4$ & $2.09 \pm 1.2$ \\
Mama izq. & $1.97 \pm 0.81$ & $2.13 \pm 1.4$ & $2.32 \pm 0.91$ \\
Esternón & $0.98 \pm 0.88$ & $1.25 \pm 1.1$ & $1.54 \pm 0.88$ \\
Ombligo & $0.79 \pm 0.94$ & $0.85 \pm 1.4$ & $1.24 \pm 0.85$ \\
\hline
\end{tabular}

TABLA 11. DP en el Plano 2: Sup. Der. con prescripción de D de 18 Gy al $80 \%$ de isodosis.

\begin{tabular}{lccc}
\hline \multicolumn{4}{c}{$80 \%$ de isodosis. } \\
\hline Plano 2: Sup. Der. & \multicolumn{3}{c}{$18 \mathrm{~Gy}$ al $80 \%$ de isodosis } \\
\hline Cono & $1.0 \mathrm{~cm}$ & $2.0 \mathrm{~cm}$ & $3.0 \mathrm{~cm}$ \\
\hline UM & 3228 & 2692 & 2524 \\
\hline Posición & \multicolumn{3}{c}{ Dosis periférica (cGy) } \\
\hline Ojo Der. & $10.88 \pm 0.87$ & $32.03 \pm 1.4$ & $66.23 \pm 0.57$ \\
Ojo Izq. & $15.92 \pm 0.99$ & $34.98 \pm 1.3$ & $50.04 \pm 0.59$ \\
Tiroides & $1.98 \pm 0.94$ & $3.88 \pm 1.3$ & $5.05 \pm 0.45$ \\
Pulmón Sup. Der. & $2.54 \pm 0.99$ & $8.98 \pm 0.63$ & $13.19 \pm 0.58$ \\
Pulmón Sup. Izq. & $1.53 \pm 0.99$ & $3.85 \pm 0.70$ & $3.88 \pm 0.48$
\end{tabular}

doi: https://doi.org/10.21754/tecnia.v28i2.343

\begin{tabular}{lccc} 
Mama der. & $0.87 \pm 0.99$ & $2.67 \pm 0.68$ & $2.74 \pm 0.52$ \\
Mama izq. & $1.14 \pm 1.2$ & $3.69 \pm 0.82$ & $3.87 \pm 1.1$ \\
Esternón & $0.97 \pm 1.3$ & $1.87 \pm 0.91$ & $2.25 \pm 1.2$ \\
Ombligo & $0.67 \pm 1.2$ & $1.47 \pm 0.82$ & $1.64 \pm 1.1$ \\
\hline
\end{tabular}

TABLA 12. DP en el Plano 2: Sup. Izq. con prescripción de D de 18 Gy al $80 \%$ de isodosis.

\begin{tabular}{lccc}
\hline Plano 2: Sup. Izq. & \multicolumn{3}{c}{$18 \mathrm{~Gy}$ al $80 \%$ de isodosis } \\
\hline Cono & $1.0 \mathrm{~cm}$ & $2.0 \mathrm{~cm}$ & $3.0 \mathrm{~cm}$ \\
\hline UM & 3224 & 2688 & 2520 \\
\hline Posición & \multicolumn{3}{c}{ Dosis periférica (cGy) } \\
\hline Ojo Der. & $13.81 \pm 1.2$ & $35.63 \pm 0.99$ & $46.79 \pm 1.4$ \\
Ojo Izq. & $20.08 \pm 1.1$ & $40.95 \pm 1.0$ & $60.91 \pm 1.6$ \\
Tiroides & $4.41 \pm 0.98$ & $5.90 \pm 1.1$ & $6.89 \pm 1.4$ \\
Pulmón Sup. Der. & $4.04 \pm 0.61$ & $5.33 \pm 0.87$ & $5.71 \pm 1.4$ \\
Pulmón Sup. Izq. & $9.72 \pm 0.67$ & $11.25 \pm 0.87$ & $13.19 \pm 1.6$ \\
Mama der. & $3.63 \pm 0.72$ & $4.72 \pm 0.87$ & $5.58 \pm 1.6$ \\
Mama izq. & $2.34 \pm 0.75$ & $3.49 \pm 0.78$ & $4.35 \pm 0.98$ \\
Esternón & $1.67 \pm 0.78$ & $2.22 \pm 0.82$ & $3.57 \pm 0.93$ \\
Ombligo & $1.13 \pm 0.75$ & $1.34 \pm 0.87$ & $1.72 \pm 0.99$ \\
\hline
\end{tabular}

Tabla 13. DP en el Plano 3: Sup. Der. con prescripción de D de 18 Gy al $80 \%$ de isodosis.

\begin{tabular}{lccc}
\hline \multicolumn{4}{c}{$80 \%$ de isodosis. } \\
\hline Plano 3: Sup. Der. & \multicolumn{3}{c}{$18 \mathrm{~Gy}$ al 80\% de isodosis } \\
\hline Cono & $1.0 \mathrm{~cm}$ & $2.0 \mathrm{~cm}$ & $3.0 \mathrm{~cm}$ \\
\hline UM & 3024 & 2548 & 2416 \\
\hline Posición & \multicolumn{3}{c}{ Dosis periférica (cGy) } \\
\hline Ojo Der. & $25.47 \pm 1.0$ & $62.80 \pm 0.85$ & $150.81 \pm 0.97$ \\
Ojo Izq. & $21.37 \pm 1.1$ & $40.67 \pm 0.88$ & $67.93 \pm 0.92$ \\
Tiroides & $4.53 \pm 1.0$ & $5.07 \pm 0.86$ & $6.95 \pm 0.97$ \\
Pulmón Sup. Der. & $6.87 \pm 1.1$ & $9.44 \pm 1.2$ & $13.41 \pm 1.0$ \\
Pulmón Sup. Izq. & $4.14 \pm 1.2$ & $4.75 \pm 1.2$ & $5.87 \pm 0.99$ \\
Mama der. & $2.97 \pm 1.1$ & $2.20 \pm 1.3$ & $3.34 \pm 1.0$ \\
Mama izq. & $3.07 \pm 0.86$ & $3.31 \pm 1.3$ & $3.88 \pm 0.87$ \\
Esternón & $2.14 \pm 0.94$ & $2.24 \pm 1.3$ & $2.69 \pm 0.83$ \\
Ombligo & $0.64 \pm 0.89$ & $1.35 \pm 1.3$ & $1.67 \pm 0.92$ \\
\hline
\end{tabular}

Tabla 14. DP en el Plano 3: Sup. Izq. con prescripción de D de 18 Gy al $80 \%$ de isodosis.

\begin{tabular}{|c|c|c|c|}
\hline \multicolumn{4}{|c|}{$80 \%$} \\
\hline Cono & $1.0 \mathrm{~cm}$ & $2.0 \mathrm{~cm}$ & $3.0 \mathrm{~cm}$ \\
\hline UM & 3032 & 2552 & 2420 \\
\hline Posición & \multicolumn{3}{|c|}{ Dosis periférica (cGy) } \\
\hline Ojo Der. & $20.35 \pm 2.0$ & $45.67 \pm 1.0$ & $84.78 \pm 1.5$ \\
\hline Ojo Izq. & $34.02 \pm 1.8$ & $53.71 \pm 7.4$ & $113.92 \pm 0.81$ \\
\hline Tiroides & $3.45 \pm 1.9$ & $4.15 \pm 0.75$ & $7.77 \pm 1.1$ \\
\hline Pulmón Sup. Der. & $2.19 \pm 0.90$ & $2.66 \pm 0.82$ & $5.25 \pm 0.95$ \\
\hline Pulmón Sup. Izq. & $3.30 \pm 1.0$ & $6.39 \pm 0.82$ & $11.71 \pm 0.87$ \\
\hline Mama der. & $1.69 \pm 1.1$ & $1.55 \pm 1.1$ & $3.13 \pm 1.2$ \\
\hline Mama izq. & $0.88 \pm 1.1$ & $1.04 \pm 1.7$ & $1.75 \pm 1.4$ \\
\hline Esternón & $0.71 \pm 1.0$ & $0.76 \pm 1.6$ & $1.58 \pm 1.1$ \\
\hline Ombligo & $0.45 \pm 1.0$ & $0.53 \pm 1.7$ & $0.87 \pm 1.1$ \\
\hline
\end{tabular}

TABLA 15. DP en el Plano 4: Centro con prescripción de D de 18 Gy al $80 \%$ de isodosis.

\begin{tabular}{lccc}
\multicolumn{4}{c}{$80 \%$ de isodosis. } \\
\hline Plano 4: Centro & \multicolumn{3}{c}{18 Gy al 80\% de isodosis } \\
\hline Cono & $1.0 \mathrm{~cm}$ & $2.0 \mathrm{~cm}$ & $3.0 \mathrm{~cm}$ \\
\hline UM & 3944 & 3252 & 3020 \\
\hline Posición & \multicolumn{3}{c}{ Dosis periférica (cGy) } \\
\hline Ojo Der. & $17.14 \pm 1.2$ & $21.15 \pm 0.77$ & $41.56 \pm 1.4$ \\
Ojo Izq. & $14.23 \pm 1.2$ & $20.74 \pm 0.79$ & $33.90 \pm 0.98$ \\
Tiroides & $8.09 \pm 1.2$ & $8.65 \pm 0.77$ & $11.07 \pm 1.2$ \\
Pulmón Sup. Der. & $7.43 \pm 0.95$ & $8.53 \pm 0.78$ & $10.09 \pm 1.2$ \\
Pulmón Sup. Izq. & $6.74 \pm 1.0$ & $7.82 \pm 0.78$ & $9.43 \pm 1.2$ \\
Mama der. & $5.68 \pm 1.2$ & $7.06 \pm 0.85$ & $9.38 \pm 1.2$ \\
Mama izq. & $4.62 \pm 0.92$ & $7.06 \pm 0.61$ & $10.66 \pm 0.79$ \\
Esternón & $2.66 \pm 0.98$ & $2.94 \pm 0.65$ & $4.11 \pm 0.79$ \\
Ombligo & $1.05 \pm 0.95$ & $1.52 \pm 0.69$ & $2.10 \pm 0.65$ \\
\hline
\end{tabular}

Revista TECNIA Vol. 28 N 1 Agosto - Diciembre 2018 
TABLA 16. DP en el Plano 4: Centro con prescripción de D de 12 Gy al $80 \%$ de isodosis.

\begin{tabular}{lccc}
\hline Plano 4: Centro & \multicolumn{3}{c}{$12 \mathrm{~Gy}$ al 80\% de isodosis } \\
\hline Cono & $1.0 \mathrm{~cm}$ & $2.0 \mathrm{~cm}$ & $3.0 \mathrm{~cm}$ \\
\hline UM & 2628 & 2168 & 2012 \\
\hline Posición & \multicolumn{3}{c}{ Dosis periférica (cGy) } \\
\hline Ojo Der. & $10.68 \pm 0.90$ & $15.21 \pm 0.62$ & $27.69 \pm 0.92$ \\
Ojo Izq. & $9.76 \pm 0.87$ & $13.77 \pm 0.77$ & $20.57 \pm 0.77$ \\
Tiroides & $4.63 \pm 0.93$ & $6.04 \pm 0.87$ & $8.63 \pm 0.80$ \\
Pulmón Sup. Der. & $2.94 \pm 0.70$ & $4.59 \pm 0.85$ & $7.37 \pm 0.78$ \\
Pulmón Sup. Izq. & $3.59 \pm 0.72$ & $5.12 \pm 0.91$ & $5.56 \pm 0.71$ \\
Mama der. & $3.15 \pm 0.75$ & $5.13 \pm 0.96$ & $5.72 \pm 0.78$ \\
Mama izq. & $3.89 \pm 0.69$ & $5.61 \pm 0.88$ & $6.45 \pm 0.84$ \\
Esternón & $2.26 \pm 0.74$ & $2.31 \pm 0.95$ & $2.42 \pm 0.79$ \\
Ombligo & $0.23 \pm 0.73$ & $0.45 \pm 0.92$ & $0.50 \pm 0.71$ \\
\hline
\end{tabular}

TABLA 17. DP en el Plano 4: Centro con prescripción de D de 12 Gy al $50 \%$ de isodosis.

\begin{tabular}{lccc} 
Plano 4: Centro & \multicolumn{3}{c}{12 Gy al $50 \%$ de isodosis } \\
\hline Cono & $1.0 \mathrm{~cm}$ & $2.0 \mathrm{~cm}$ & $3.0 \mathrm{~cm}$ \\
\hline UM & 4208 & 3468 & 3220 \\
\hline Posición & \multicolumn{3}{c}{ Dosis periférica (cGy) } \\
\hline Ojo Der. & $18.30 \pm 0.91$ & $23.98 \pm 1.5$ & $31.47 \pm 0.91$ \\
Ojo Izq. & $19.02 \pm 1.1$ & $22.55 \pm 1.6$ & $28.64 \pm 0.97$ \\
Tiroides & $9.95 \pm 1.0$ & $10.84 \pm 1.6$ & $12.07 \pm 0.87$ \\
Pulmón Sup. Der. & $8.29 \pm 1.1$ & $9.03 \pm 0.60$ & $10.65 \pm 0.94$ \\
Pulmón Sup. Izq. & $7.72 \pm 1.1$ & $8.35 \pm 0.62$ & $8.73 \pm 0.99$ \\
Mama der. & $4.21 \pm 1.1$ & $8.27 \pm 0.65$ & $8.53 \pm 1.0$ \\
Mama izq. & $5.23 \pm 0.77$ & $8.83 \pm 0.72$ & $10.94 \pm 1.4$ \\
Esternón & $3.19 \pm 0.95$ & $3.46 \pm 0.71$ & $3.60 \pm 1.5$ \\
Ombligo & $2.66 \pm 1.1$ & $2.99 \pm 0.62$ & $3.12 \pm 1.5$ \\
\hline
\end{tabular}

\section{CONCLUSIONES}

Del análisis de la DP en los 4 planos, se puede observar que en el Plano 1 la DP aumenta conforme aumenta el tamaño de colimador. Es así que en el ojo derecho se registra más DP en el lado Sup. Izq., de (6. 19 $\pm 0.36)$ cGy a (17.09 \pm 0.51$)$ cGy, para colimadores de $1 \mathrm{a}$ $3 \mathrm{~cm}$ respectivamente; en el ojo izquierdo se registra más DP en el lado Sup. Der., de (12.38 \pm 0.85$)$ cGy a (30.31 \pm 0.92) cGy, para colimadores de 1 a $3 \mathrm{~cm}$ respectivamente; en la tiroides se registra mayor DP en los lados inferiores, para el lado Inf. Der., de (4.39 \pm $0.64)$ cGy a (17.91 \pm 1.3$)$ cGy, para colimadores de 1 a 3.0 $\mathrm{cm}$ respectivamente y para el lado Inf. Izq. de (5.21 \pm 1.1$)$ cGy a (18.89 \pm 0.70$)$ cGy, para colimadores de 1 a 3 cm respectivamente; en el pulmón Sup. Der. se registra más DP en el lado Inf. Der., de $(6.63 \pm 1.0)$ cGy a $(21.49 \pm 1.2)$ CGy, para colimadores de 1 a $3 \mathrm{~cm}$ respectivamente y en el pulmón Sup. Izq. se registra más DP en el lado Inf. Izq., de $(4.09 \pm 1.3)$ cGy a $(21.65 \pm 0.61)$ CGy, para colimadores de 1 a $3 \mathrm{~cm}$ respectivamente.

Del Plano 2, de igual manera, se observa que la DP aumenta conforme aumenta el tamaño de colimador, en el ojo derecho se registra más DP en el lado Sup. Der., de $(10.88 \pm 0.87)$ cGy a $(66.23 \pm 0.57)$ cGy, para colimadores de 1 a $3 \mathrm{~cm}$ respectivamente; en el ojo izquierdo se registra más DP en el lado Sup. Izq., de $(20.08 \pm 1.1)$ cGy a $(60.91 \pm 1.6)$ cGy, para colimadores de 1.0 a $3.0 \mathrm{~cm}$ respectivamente y en la tiroides se registra mayor $D$ en los lados inferiores, registrándose mayor DP en el lado Inf. Izq. de $(7.10 \pm 1.1)$ cGy a $(27.14 \pm 1.0)$ cGy, para colimadores de 1 a $3 \mathrm{~cm}$ respectivamente.

En el Plano 3 el comportamiento es similar con respecto a los dos planos anteriores, en el ojo derecho se registra más DP en el lado Sup. Der., de (25.47 \pm 1.0$)$ cGy a (150.81 \pm 0.97$)$ cGy, para colimadores de 1.0 a 3.0 $\mathrm{cm}$ respectivamente; en el ojo izquierdo se registra más DP en el lado Sup. Izq., de (34.02 \pm 1.8$)$ cGy a (113.92 \pm $0.81)$ CGy, para colimadores de 1 a $3 \mathrm{~cm}$ respectivamente y en la tiroides se registra mayor $D$ en el lado Sup. Izq. con una DP de (7.77 \pm 1.1$)$ cGy para el colimador de 3.0 $\mathrm{cm}$. Como el Plano 3 está más lejano de la tiroides la DP es menor comparado con la DP en los Planos 1 y 2.

Por último, para el Plano 4 se analizó la influencia de la prescripción de DP con respecto al tamaño de colimador. De esta forma se puede observar que en el ojo derecho hay mayor DP en la prescripción de $18 \mathrm{~Gy}$ al $80 \%$ de isodosis, de $(17.14 \pm 1.2)$ cGy a $(41.56 \pm 1.4)$ cGy, para colimadores de 1 a $3 \mathrm{~cm}$ respectivamente; en el ojo izquierdo hay mayor DP en la prescripción de $18 \mathrm{~Gy}$ al $80 \%$ de isodosis, de $(14.23 \pm 1.2)$ cGy a $(33.90 \pm 0.98)$ cGy, para colimadores de 1 a $3 \mathrm{~cm}$ respectivamente. Sin embargo, en la tiroides hay mayor DP en la prescripción de 12 Gy al 50\% de isodosis, de (9.95 \pm 1.0$)$ cGy a (12.07 \pm 0.87) cGy, para colimadores de 1 a $3 \mathrm{~cm}$ respectivamente.

Los resultados son comparables con el de Maria de Salete et al. [19], ellos estimaron la DP en ojos, tiroides, zona torácica y pelvis en pacientes que recibieron radiocirugía en un linac de $6 \mathrm{MV}$. Se evaluaron 11 pacientes y se estimó la DP con dosímetros termoluminiscentes. Encontrando resultados de $D$ promedio en el ojo derecho de $(4.8 \pm 2.6)$ cGy, ojo izquierdo $(6.5 \pm 7.8)$ cGy, tiroides $(4.2 \pm 2.8)$ cGy, zona torácica (1.6 \pm 0.9$)$ cGy y pelvis (0.4 \pm 0.3$)$ cGy. Concluyendo, que aunque la $D$ no excede los límites de tolerancia en los ojos, que pueda provocar su opacidad, es necesario que el riesgo asociado con la DP sean tomados en cuenta por radioterapeutas en la planificación de los procedimientos.

Se concluye que la DP reproduce una dependencia proporcional con respecto a las unidades de monitor, debido a que hay un incremento en la radiación por fuga y dispersión por cabezal del acelerador al aumentar las unidades de monitor. Así mismo, mientras más grande el colimador mayor es la DP, es así que se encontró mayor DP en el colimador de $3 \mathrm{~cm}$ en el Plano 3: Sup. Der. con un $6.7 \%$ de una D prescrita de 18 Gy al $80 \%$ de isodosis en la posición anatómica del ojo derecho del fantoma.

Por otro lado, a mayor distancia del isocentro, la DP disminuye. Muestra de ello es la mayor DP registrada en el Plano 2: Inf. Izq., con un $1.2 \%$ de la $D$ prescrita en la tiroides con una distancia cráneo caudal de $9.4 \mathrm{~cm}$ con respecto al blanco y de $0.09 \%$ de la $D$ prescrita en el ombligo con una distancia cráneo caudal de $42.4 \mathrm{~cm}$ con 
respecto al blanco, ambos para el colimador de $3 \mathrm{~cm}$ y $D$ prescrita de $18 \mathrm{~Gy}$ al $80 \%$ de isodosis.

Se concluye mediante este trabajo que se puede optimizar la planificación de los tratamientos en radiocirugía evitando o minimizando haces de salida hacia el cristalino, tiroides, y pecho.

Además se recomienda que para realizar la optimización durante el procedimiento en radiocirugía se tiene que conocer la dependencia de la DP con respecto a la localización del blanco, el tamaño de colimador y la distancia.

\section{AGRADECIMIENTOS}

Al Instituto de radiocirugía del Perú (INRAPE), por facilitarme los equipos y materiales necesarios para llevar a cabo todas las actividades propuestas durante el desarrollo de esta tesis. A la clínica San Pablo por facilitarme el uso de sus instalaciones, así como del equipo necesario.

\section{REFERENCIAS}

[1] Di Betta E, Fariselli L, Bergantin A, Locatelli F, Del Vecchio A, Broggi S and Fumagalli M 2010 Med. Phys. 37(7), 3587-3594

[2] Xu XG, Badnarz B, Paganetti L 2008 Phys. Med. Biol. 53(13), 193-241

[3] Bonato C, Dias H, Alves M, Duarte L, Dias T, Dalenogare M, Viegas C, Elnecave R 2014 Radiat. Oncol. 9, 40

[4] Maarouf M, Treuer H, Kocher M, Voges J, Gierich A, Sturm V 2005 Strahlentherapie Onkol. 181(7), 463-467

[5] Brenner J et al. 2003 Proc. Natl. Acad. Sci. U.S.A. 100(24), 1376113766

[6] Ashland 2018 Recuperado de: http://www.gafchromic.com/gafchromic-film/radiotherapyfilms/EBT/index.asp

[7] ICRP Publication 892002 Pergamon 32(3-4)

[8] Webb S 1993 The Physics of Tridimensional Radiation Therapy. Conformal Radiotherapy, Radiosurgery and Treatment Planning Institute of Physics; London

[9] Podgorsak EB 2005 Radiation Oncology Physics: A Handbook for Teachers and Student IAEA; Austria

[10] Arráns R et al. 2009 Rev. Fis. Med. 10,83-104

[11] Adrada A 20011 Msc. tesis, Universidad Nacional de Cuyo

[12] Williams M, Metcalfe P 2011 Radiochromic film dosimetry and its applications in radiotherapy, proceedings of Concepts and Trends in Medical Radiation Dosimetry University of Wolongong; Australia

[13] Seiko Epson Corporation 2018 Recuperado de: https://www.epson.es/products/scanners/businessscanners/expression-11000xl

[14] Devic S, Aldelaijan S, Mohammed H, Tomic N, Liang LH, DeBlois F, Seuntjens J 2010 Med. Phys. 37(5), 2208- 2214

[15] Maayers S 2011 Msc. thesis, University of Wollongong

[16] Devic S, Seuntjens J, Sham E, Podgorask E, Ross C, Kirov A, Soares C 2005 Med. Phys. 32(7), 2245-2253

[17] Carestream Health 2018 Recuperado de: https://www.carestream.com/es/es/medical/products/radiograp hy/film-systems/film-solutions-for-oncology

[18] Nieva S 2013 Slide Share Recuperado de: https://es.slideshare.net/silvianievaramos/tema-o-dca.

[19] De Salete M, Jamil H, Azevedo S 2012 Radiol. Bras. 45(2), 83-86
Los artículos publicados por TECNIA pueden ser compartidos a través de la licencia Creative Commons: CC BY 4.0 Perú. Permisos lejos de este alcance pueden ser consultados a través del correo revistas@uni.edu.pe 